\title{
Research Article \\ Genetic Algorithm for Multiuser Discrete Network Design Problem under Demand Uncertainty
}

\author{
Wu Juan, ${ }^{1,2}$ Lu Huapu, ${ }^{1}$ Yu Xinxin, ${ }^{3}$ and Bian Changzhi ${ }^{4}$ \\ ${ }^{1}$ Institute of Transportation Engineering, Tsinghua University, Beijing 100084, China \\ ${ }^{2}$ Academy of Military Transportation, Tianjin 300161, China \\ ${ }^{3}$ Transport Planning and Research Institute, Ministry of Transport, Beijing 100028, China \\ ${ }^{4}$ China Academy of Urban Planning and Design, Beijing 100044, China
}

Correspondence should be addressed to Wu Juan, wujuan_qh@yahoo.cn

Received 6 September 2012; Accepted 29 October 2012

Academic Editor: Baozhen Yao

Copyright (c) 2012 Wu Juan et al. This is an open access article distributed under the Creative Commons Attribution License, which permits unrestricted use, distribution, and reproduction in any medium, provided the original work is properly cited.

Discrete network design is an important part of urban transportation planning. The purpose of this paper is to present a bilevel model for discrete network design. The upper-level model aims to minimize the total travel time under a stochastic demand to design a discrete network. In the lower-level model, demands are assigned to the network through a multiuser traffic equilibrium assignment. Generally, discrete network could affect path selections of demands, while the results of the multiuser traffic equilibrium assignment need to reconstruct a new discrete network. An iterative approach including an improved genetic algorithm and Frank-Wolfe algorithm is used to solve the bi-level model. The numerical results on Nguyen Dupuis network show that the model and the related algorithms were effective for discrete network design.

\section{Introduction}

With the development of cities, travel demand is high and widely spread. The capacity of the current transport system remains limited to accommodate the increasing demand. It has emerged as an important area for progress in handling effective transport planning, in which some new links or roadway segments are added to expanding the current system capacity. The discrete network design problem (DNDP) deals with the selection of link additions to an existing road network, with a given demand from each origin to each destination. The objective of DNDP is often to optimize a given system performance measure such as to minimize total system travel cost, while accounting for the route choice behaviors of network users. Farvaresh and Sepehri [1] presented a single-level mixed integer linear formulation for discrete network design. Miandoabchi and Farahani [2] presented a discrete network design 
model, in which the concurrent design of street capacity, street direction, and lane allocations for two-way street are optimized based on the reserve capacity maximization. In traditional transportation network design, the travel demand is often assumed as a constant and users are assumed to belong to a single class. However, the assumptions are inappropriate to reallife applications.

Different forms may be adopted for different aims in those literatures; but uncertainty in decision making and the diversity of the users are not considered. This paper seeks to make two contributions to the literature. Firstly, when the travel demand is uncertainty, uncertain optimization theory will be used. Secondly, when the users are diversity, they will be classified and treated, respectively. Therefore, a bi-level model was proposed for traffic network design. The upper-level model takes the travel time as the main consideration factor to optimize the network design model. Considering the fact that the travel demand changes along with the change of the network, the lower-level model assigns the users again in the network optimized by the upper-level model.

The uncertain transportation network design model based on the stochastic programming theory assumes that travel demand is a stochastic variable submitting to a known probability distribution. At the same time, the optimal network plan will be obtained with stochastic bi-level programming model. Patriksson [3] considered demand uncertainty in stochastic bi-level programming model, in which the upper-level model is to minimize expected value of the objective function, and the lower-level model is the equilibrium conditions of variation inequality.

Ukkusuri and Waller [4] provided the chance constrained programming model and two-phase compensation stochastic programming model for designing the transportation network with a single end and uncertain OD demand. When traffic flow meets the dynamic user equilibrium condition, cell transmission model (CTM) can be used, and the numerical calculation shows that the suboptimal solutions will be obtained without considering the uncertainty of demand. Karoonsoontawong and Waller [5] established a continuous transportation network design model under the demand uncertainty. Assume that every demand situation meets a dynamic user equilibrium to describe the traffic flow based on the CTM model of Daganzo [6]. The model minimizes the weighted average of the expected mean value and the expected risk to improve model robustness against demand uncertainty. Yang [7] analyzed the behavior of equilibrium flows with elastic demand which can be used to measure the demand and performance characteristics of the transportation networks. Li et al. [8] attempted to present road toll design model for congested road networks with uncertain demand. A heuristic algorithm based on the sample average approximation approach and a sensitivity analysis is used to solve the network design model.

There are two types of methods dealing with multiuser problem in transportation network design problem. One method is to classify users according to traffic mode characteristics and vehicle types; then each category of users has different cost functions. Similar study has been done by Smith [9]; and so forth. Another method assumes that vehicle types of travelers are the same and have the same effect on traffic flow; but there are differences in time value. According to the different way of study, time value distribution can be assumed as discrete or continuous, corresponding to limited categories of users or infinite categories of users. Research about multiuser network equilibrium based on discrete time value has been done by Daganzo [6], Yang and Zhang [10]. Research about multiuser network equilibrium based on continuous time value has been done by Dial [11].

In order to correct the inappropriate assumptions in traditional transportation network design, in this paper, the OD trip demand elements are supposed as stochastic variables 
submitting to given probability distribution. Travelers are divided into different groups by the value of time and a novel multiuser network design model is established. However, for network design, it is difficult to be solved through classical optimization techniques [12]. Recently many studies have proved that heuristic algorithms are suitable for large-scale transit network optimization problems, such as ant colony algorithm [13-15] and simulated annealing algorithm [16].

Genetic algorithm (GA) is a search heuristic that based on the idea extracting from the process of natural evolution. Recently many studies have proved that genetic algorithm is suitable for solving network design problem. Pattnaik et al. [17] presented a GA-based optimization method to design transit network, in which the total cost of user and operator was to be minimized. Agrawal and Mathew [12] presented an optimization model for transit network. The model was aiming to minimize the total system cost which included the operating cost and the generalized travel cost. Bielli et al. [18] developed a heuristic based on GA to design transit network. In the heuristic, a multicriteria analysis was used to estimate the fitness values. Thus, in this paper, GA is also used to solve our discrete network design problem.

This paper has been organized in the following way. Section 2 describes the travelers time value and multiuser classification; Section 3 is about the optimization model, including the problem formulations and the basic notations of variables; Section 4 describes genetic algorithm and Frank-Wolfe algorithm for discrete network design problem. Numerical analysis is carried out in Section 5, and lastly, the conclusions are drawn in Section 6.

\section{Analysis of the Fundamental Factors}

\subsection{Traveler Time Value}

In economics, social activities can be abstracted into production and consumption behavior. The elements to describe different activities are often different, and time consumption is often used to measure the activity efficiency. Time as a resource, its value should be reasonable measured for better and efficient allocation. Time value represents time saving in terms of money. Under a given space-time environment, the factors that affect time value mainly include traveler characteristics, travel purpose, transportation modes, and other aspects.

In different conditions, the influence degree of each factor is different. Evaluation of traveler time value is a comprehensive reflection of these factors. The following is the introduction of the main factors that affect traveler time value.

\section{(1) Traveler Characteristics}

Different social and economic characteristics often affect traveler behavior. The income level is the greatest effect among their characteristics. High-income passengers pay more attention to quickness, comfort, safety, and service level than the travel fee while low-income passengers tend to use more time to save money.

\section{(2) Travel Purpose}

Travel purpose is the motive of a trip. When travelers are confronted with different travel purposes, there are often different choices for them to select and different choices with different time and cost. For example, a trip for work has time constraint while a trip for 
shopping has more free time; so the time value for work is more than the time value for shopping. In some special circumstances, such as the traffic accidents or emergency which need medical assistance, time value is much higher than that of normal work trip.

\section{(3) Transportation Mode}

Traveler time value is not only related to travel purpose, but also transportation mode. Different transportation modes have different speeds, convenience, and comfort, and those differences affect travelers to select different transportation modes. When travelers choose some transportation modes, they often consider those factors like travel time, travel cost, and the auxiliary or additional travel time. For example, car can provide prompt door to door service, so its time value is high. The bus needs more time not only aboard bus but also out of bus for waiting and walking; so its time value is low.

\section{(4) Other Factors}

In addition to the influences of the above factors on traveler time value, travel distance, road traffic conditions, vehicles conditions, and the service level [19], to a certain degree, also have influences on the evaluation of travelers time value.

\subsection{User Classification}

There are two types of methods dealing with multiuser problem in transportation network. One method is to classify users according to transportation modes and vehicle types, in which each category of users has its cost function. In this paper, for the convenience of the study, all the traveler time values are also categorized into two kinds: discrete and continuous. In the same way, the users are considered as two kinds: limited categories of users and infinite categories. This paper assumes that the difference among transportation network users is the traveler time value, and the other characteristic is not considered.

\section{Multiuser Discrete Network Design Model under OD Demand Uncertainty}

\subsection{Basic Notations}

The following are the notations used in the model formulation.

$N$ : Transportation network nodes set

A: Transportation network links set

$O_{r}$ : The trip generation flow from the terminal $r$

$D_{s}$ : The trip attraction flow from the terminal $s$

$P_{r s}$ : The routes set from the origin terminal $r$ to the destination terminal $s$

$x_{a}$ : Traffic flow on link $a$

$t_{a}(\mathbf{x})$ : Travel time impedance function of link $a$

$f_{k}^{r s}$ : Traffic flow on path $k$ between OD pair $r$ and $s$ 
$c_{k}^{r s}$ : Traffic cost on path $k$ between OD pair $r$ and $s$

$\delta_{a, k}^{r s}$ : When $a$ belongs to path $k$ which is between OD pair $r$ and $s, \delta=1$; otherwise $\delta=0$

$\bar{A}$ : Set of new built or expanded links

$y_{a}$ : Decision variable of link $a, y_{a} \in\{0,1\}$, when link will be new built or expanded, $y_{a}=1$; otherwise, $y_{a}=0$

$C_{a}$ : Transport capacity of link $a$

$L_{a}$ : Length of link $a$

$G_{a}\left(y_{a}\right)$ : Cost of new built or expanded link $a$

$B$ : Budget of all the new built or expanded links

$\Omega$ : All possible scenarios set of uncertain travel demand

$\omega$ : Any realization of uncertain travel demand

$p^{\omega}$ : Realization probability of uncertain travel demand scenario $w$

$\rho$ : Mean and variance weight of travel time given by planning decision makers.

\subsection{Multiuser Assumption}

Each group of travelers has similar social and economic characteristics (such as income level). If travelers can be divided into discrete $I$ groups according to time value, the time value of travelers $i$ from the origin terminal $r$ to the destination terminal $s$ is set to $g_{r s}^{i}$ when $i \in I$. Thus, the user cost includes two parts. One is the cost of travel time value which is related to route flow; the other is the toll fee $\tau_{a}$, which is a constant. The sum of the cost from two parts is changed as the variation of the travel time value. Formula (3.1) is the generalized cost of the use of link a by $i$ group users. Formula (3.2) is the time cost on path $k$ between OD pair $r$ and s. Formula (3.3) is the expenses cost on path $k$ between OD pair $r$ ands. Formula (3.4) generalized the cost of the use of path $k$ between OD pair $r$ and $s$ by $i$ group users:

$$
\begin{gathered}
g_{a}^{i}\left(x_{a}\right)=t_{a}\left(x_{a}\right)+\frac{1}{\psi_{i}} \tau_{a}, \quad \forall a \in A, i \in I, \\
c_{k}^{r s}=\sum t_{a}\left(x_{a}\right) \delta_{a, k}^{r s}, \quad \forall r \in R, s \in S, k \in K, \\
\tau_{k}^{r s}=\sum \tau_{a} \delta_{a, k}^{r s}, \quad \forall r \in R, s \in S, k \in K, \\
g_{k, i}^{r s}=c_{k}^{r s}+\frac{1}{\psi_{i}} \tau_{k}^{r s}, \quad \forall r \in R, s \in S, k \in P_{r s}, i \in I .
\end{gathered}
$$

\subsection{Multiuser Network Optimization Model under OD Demand Uncertainty}

OD trip demand of each group travelers is supposed as a random variable submitting to the given probability distribution. In practical calculation, when Monte-Carlo random sampling is used to form a demand scenario set $\Omega$, any demand scenario realization is $w$. OD trip demand is $q_{r s}^{i, \omega}$, where $i$ is the set of groups of travelers. Scenario realization probability is $p^{\omega}$. Multiuser discrete network design model under OD trip demand uncertainty includes upperlevel model (3.5) and lower-level model (3.6), which are correlated by network improved decision variable $y$ and traffic flow $x$. 
The upper-level model (3.5) is to minimize the system total travel time mean and standard deviation with the random demand in all scenarios realization condition, when planners choose new built and rebuilt links under the capital budget constraints. The lowerlevel model (3.6) is the corresponding multiuser equilibrium of each demand scenario under the improved decisions conditions decided by the upper-level model. On has

$$
\begin{aligned}
& \min \quad Z(\mathbf{x}, \mathbf{y})=\rho \sum_{\omega} p^{\omega}\left[\sum_{a} x_{a}^{\omega} t_{a}^{\omega}\left(x_{a}^{\omega}, y_{a}\right)\right]+(1-\rho) \\
& \times\left[\sum_{\omega} p^{\omega}\left\{\sum_{a} x_{a}^{\omega} t_{a}^{\omega}\left(x_{a}^{\omega}, y_{a}\right)-\sum_{\omega} p^{\omega}\left[\sum_{a} x_{a}^{\omega} t_{a}^{\omega}\left(x_{a}^{\omega}, y_{a}\right)\right]\right\}^{2}\right]^{1 / 2} \\
& y_{a} \in\{0,1\}, \quad \forall a \in \bar{A},
\end{aligned}
$$

where $x=x(y)$ is implicit function of $y$, decided by lower-level model (3.6). On has

$$
\begin{array}{ll}
\min & T(\mathbf{x})=\sum_{a} \int_{0}^{x_{a}^{\omega}} t_{a}^{\omega}(w) d w+\sum_{a} \sum_{i \in I} \frac{1}{\psi_{i}} x_{a}^{i, \omega} \tau_{a} \\
\text { s.t. } \quad \sum_{k \in P_{r s}} f_{k, i}^{r s, \omega}=q_{r s}^{i, \omega}, \quad \forall r \in R, s \in S, \forall i \in I, \omega \in \Omega \\
f_{k, i}^{r s, \omega} \geq 0, \quad \forall r \in R, s \in S, k \in P_{r s}, i \in I, \omega \in \Omega \\
x_{a}^{i, \omega}=\sum_{r \in R} \sum_{s \in S} \sum_{k \in P_{r s}} f_{k, i}^{r s, \omega} \delta_{a k}^{r s, \omega}, \quad \forall a, i \in I, \omega \in \Omega \\
x_{a}^{\omega}=\sum_{i \in I} x_{a}^{i, \omega}, \quad \forall a, \omega \in \Omega .
\end{array}
$$

Here, travel time of link $a$ is described as BPR function.

The right balance between the mean and standard deviation is kept by weight factor $\rho(\rho \in[0,1])$, where $\rho$ shows the prediction of the planners for the average performance of uncertainty and the discrete degree of depart from the average performance. The bigger $\rho$ value is, the less the planners would like to select.

In this paper, the multiuser discrete network design problem under demand uncertainty can be shown in Figure 1. 


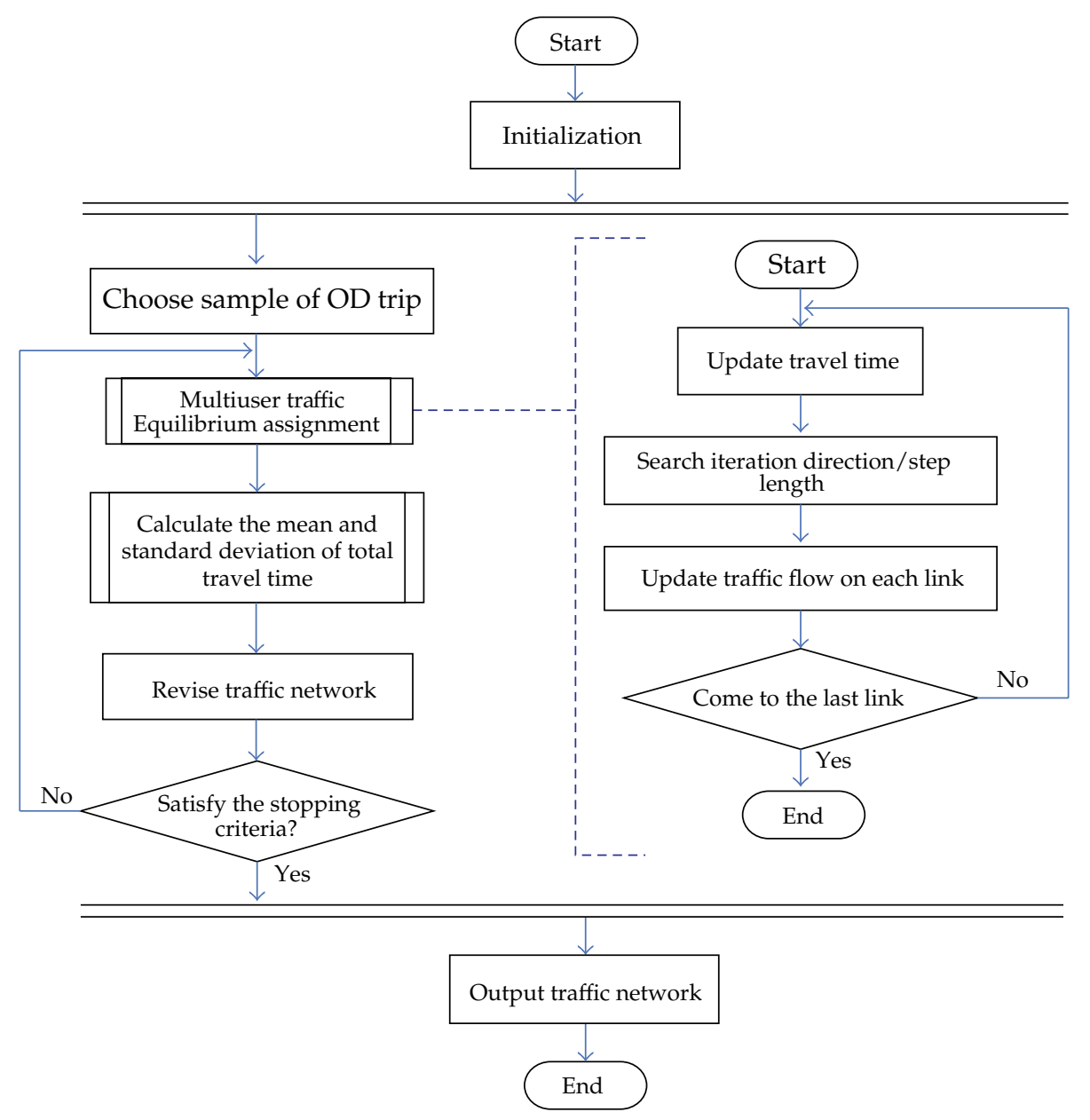

Figure 1: The optimized process of discrete network design problem.

\section{Solution Approach}

\subsection{Multiuser Traffic Equilibrium Assignment}

Using Frank-Wolfe method to solve multiuser equilibrium model consists of the following steps.

Step 1. Initialization: according to $\left\{t_{a}^{0}=t_{a}(0)\right\}$ and $\left\{\tau_{a}\right\}, 0-1$ traffic assignment based on generalized cost is conducted to each group of users demand $\left\{q_{r s}^{i}\right\}$ when $n=1$.

Step 2. Updating travel time on each link: determining generalized cost $g_{a}^{i n}\left(x_{a}^{n}\right)$ of each group of users on each link when $t_{a}^{n}=t_{a}\left(x_{a}^{n}\right)$, for all $a$.

Step 3. Searching for iterative direction: conduct 0-1 traffic assignment according to generalized cost $\left\{g_{a}^{i n}\left(x_{a}^{n}\right)\right\}$ to each group of users and get a set of additional traffic flow $\left\{y_{a}^{i n}\right\}$. 


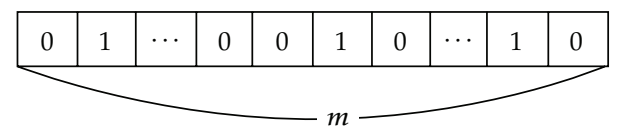

Figure 2

Step 4. Searching for iteration step length $\lambda_{n}$ based on $\min _{0 \leq \lambda_{n} \leq 1} T\left(\mathbf{x}^{\text {in }}+\lambda_{n}\left(\mathbf{y}^{\text {in }}-\mathbf{x}^{\text {in }}\right)\right)$ : to seek $\lambda_{n}$ until it meets $\min _{0 \leq \lambda_{n} \leq 1} T\left(\mathbf{x}^{i n}+\lambda_{n}\left(\mathbf{y}^{i n}-\mathbf{x}^{i n}\right)\right)$.

Step 5. Updating road traffic flow on each link:

$$
x_{a}^{i(n+1)}=x_{a}^{i n}+\lambda_{n}\left(y_{a}^{i n}-x_{a}^{i n}\right), \quad \forall a, i ; \quad x_{a}^{n+1}=x_{a}^{n}+\lambda_{n}\left(y_{a}^{n}-x_{a}^{n}\right), \quad \forall a
$$

Step 6. Convergence test: if $\left\{x_{a}^{n+1}\right\}$ already meets specified convergence criteria, calculation stops and $\left\{x_{a}^{n+1}\right\}$ are the balance solutions. Otherwise update $n=n+1$, return to Step 2 .

\subsection{GA for Multiuser Discrete Transportation Network Design}

GA is inspired by evolutionary biology like inheritance, selection, crossover, and mutation. Based on a fitness function, GA attempts to retain relatively good genetic information from generation to generation. GA has been used for solving approximately combinatorial optimization problems [20]. In this paper, GA is adopted to solve multiuser discrete transportation network design model under demand uncertainty. The following is the steps of GA.

\section{(1) Encoding}

For a discrete network design, the added links or expanded links are to assign to the current network. Thus, an integer-coded scheme is selected to represent the alternative links in this paper, and a chromosome example is as shown in Figure 2, where " 0 " represents the corresponding link remaining the current situation while " 1 " represents the links need to add new links or be expanded. For example, the chromosome is 1100110000 which represents that route no. 1, 2, 5, and 6 needs to add new links or be expanded. The other routes remain the current situation.

\section{(2) Fitness Function}

Generally, GA is optimal searching method to find the maximum fitness of the individual chromosome. Therefore, a constant $Q$ is introduced to transform our objective function to a maximum fitness function and the chromosomes are evaluated as follows:

$$
M(f)=\frac{Q}{Z}
$$

where $M(f)$ is the fitness function and $Q$ is a constant. 


\section{(3) Selection}

The basic part of the selection process is to stochastically select from one generation to create the basis of the next generation. The requirement is that the fittest individuals have a greater chance of survival than weaker ones. That is, the better the chromosomes are, the more chances to be selected they have. Therefore, the Roulette wheel selection method is used for the selection of chromosomes in this paper. In addition, to increase the performance of GA, elitism is used for selection. That is, if the elitism parameter was set to $R$; then the top $R$ chromosomes in the population are copied to the next generation.

\section{(4) Crossover}

Crossover is a genetic operator that exchanges genetic information between two parents' chromosomes to produce two new children chromosomes. The crossover operator occurs during evolution according to a given crossover rate $p_{c}$. In this paper, in crossover operation, the two links are selected, based on a simple arithmetic crossover [20], from the parent chromosomes and exchange the two links, and then generate two new children chromosomes:

$$
\begin{aligned}
& \operatorname{gen}_{k, I}^{t}=\alpha_{i} \operatorname{gen}_{k, I}^{t-1}+\left(1-\alpha_{k}\right) \operatorname{gen}_{k, I I}^{t-1}, \\
& \operatorname{gen}_{k, I I}^{t}=\alpha_{i} \operatorname{gen}_{k, I I}^{t-1}+\left(1-\alpha_{k}\right) \operatorname{gen}_{k, I}^{t-1},
\end{aligned}
$$

where gen ${ }_{k, I}^{t-1} \operatorname{gen}_{k, I I}^{t-1}$ is a pair of "parent" chromosomes; gen ${ }_{k, I}^{t} \operatorname{gen}_{k, I I}^{t}$ is a pair of "children" chromosomes; $\alpha_{k}$ is a random number between $(0,1) ; k \in[1,2,3]$ ( $k$ is the total genes for the crossover operation).

\section{(5) Mutation}

Like the crossover, the mutation operator is also associated with a mutation rate $\left(P_{m}\right)$ to determine whether or not the mutation operator is to be applied to the chromosome. An arithmetic mutation like the crossover is designed, and then a new offspring chromosome is acquired by mutation operator.

Assume a chromosome is $G=\left(\operatorname{gen}_{1}^{t}, \ldots, \operatorname{gen}_{k}^{t}, \ldots, \operatorname{gen}_{m}^{t}\right)$, if the gen ${ }_{2}^{t}$ was selected for the mutation, the mutation can be shown in (4.4):

$$
\begin{aligned}
& G^{\prime}=\left(\operatorname{gen}_{1}^{t-1}, \ldots, \operatorname{gen}_{k}^{t}, \ldots, \operatorname{gen}_{m}^{t-1}\right), \\
& \operatorname{gen}_{k}^{t}= \begin{cases}\operatorname{gen}_{k}^{t-1}+\Delta\left(t, \operatorname{gen}_{k \max }^{t}-\operatorname{gen}_{k}^{t-1}\right), & \text { if } \operatorname{random}(0,1)=0, \\
\operatorname{gen}_{k}^{t-1}+\Delta\left(t, \operatorname{gen}_{k}^{t-1}-\operatorname{gen}_{k \min }^{t}\right), & \text { if } \operatorname{random}(0,1)=1 .\end{cases}
\end{aligned}
$$

The function $\Delta(t, y)$ returns a value between $[0, y]$ given in (4.5).

$$
\Delta(t, y)=y \times\left(1-r^{\left(1-t / T_{\max }\right)^{\curlywedge}}\right)
$$




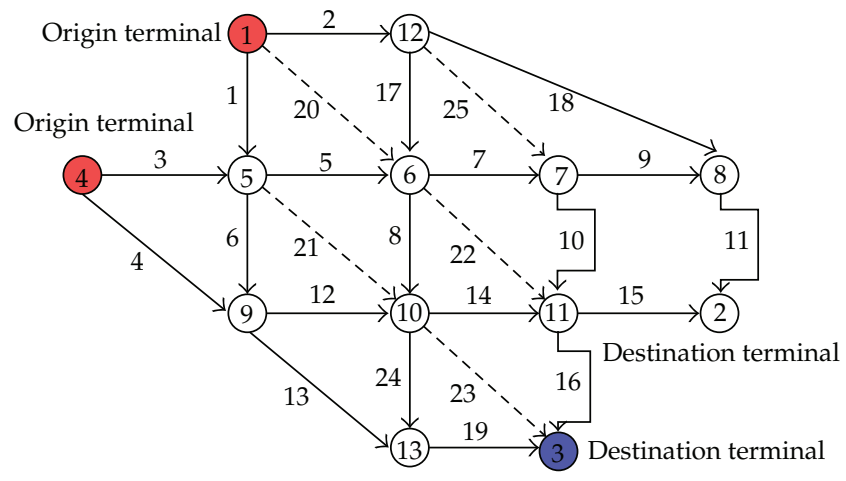

Figure 3: The networks of Nguyen Dupuis.

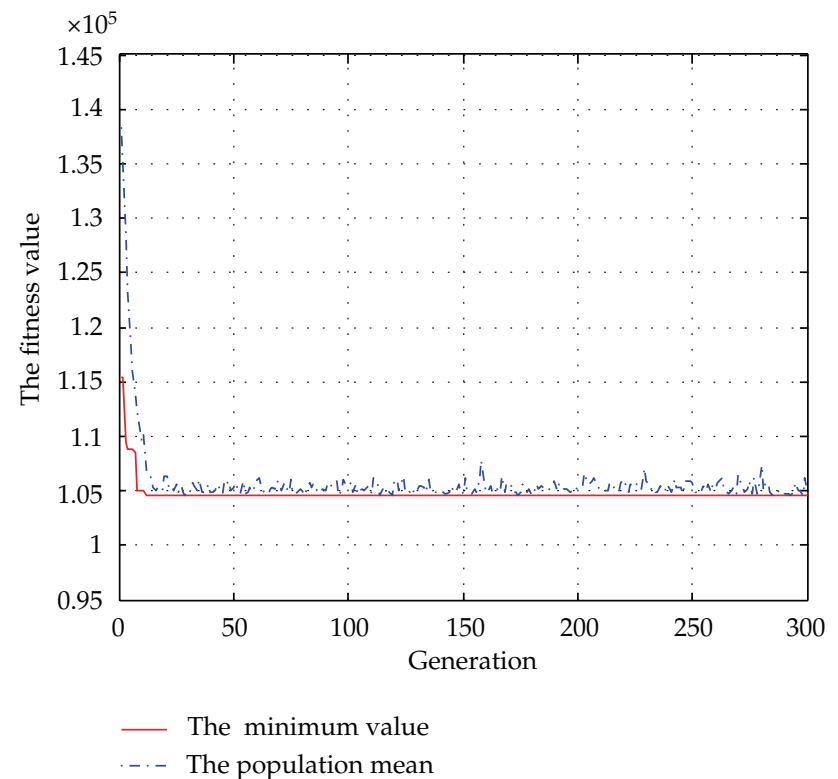

Figure 4: The solution of scenario I.

where $r$ is a random number between $[0,1] ; T_{\max }$ is the maximum number of generations; here $\mathcal{\lambda}=3$. This property causes this operation to make a uniform search in the initial space when $t$ is small and a very local one in later stages.

\section{Case Studies}

\subsection{Network Structure}

In this paper, the test network of Nguyen and Dupuis [21] is used as a case study. This network has 13 nodes, 19 links, and 4 OD pairs. The basic structures of this network is shown in Figure 3, in which a red node is the symbol for a travel demand generation point, a blue node is the symbol for a travel demand attract point, a solid line is the symbol for 
Table 1: The attribute of the Nguyen Dupuis network.

\begin{tabular}{|c|c|c|c|c|}
\hline Link & Free flow time & Existing capacity & Planning capacity & Construction cos \\
\hline 1 & 12 & 250 & 500 & 100 \\
\hline 2 & 12 & 250 & 500 & 100 \\
\hline 3 & 12 & 250 & 500 & 100 \\
\hline 4 & 24 & 150 & 250 & 100 \\
\hline 5 & 12 & 250 & 500 & 100 \\
\hline 6 & 12 & 250 & 500 & 100 \\
\hline 7 & 12 & 250 & 500 & 100 \\
\hline 8 & 12 & 250 & 500 & 100 \\
\hline 9 & 12 & 250 & 500 & 100 \\
\hline 10 & 12 & 250 & 500 & 100 \\
\hline 11 & 12 & 250 & 500 & 100 \\
\hline 12 & 12 & 250 & 500 & 100 \\
\hline 13 & 24 & 150 & 250 & 100 \\
\hline 14 & 12 & 250 & 500 & 100 \\
\hline 15 & 12 & 250 & 500 & 100 \\
\hline 16 & 12 & 250 & 500 & 100 \\
\hline 17 & 12 & 250 & 500 & 100 \\
\hline 18 & 36 & 150 & 250 & 100 \\
\hline 19 & 12 & 250 & 500 & 100 \\
\hline 20 & 24 & 0 & 250 & 100 \\
\hline 21 & 24 & 0 & 250 & 100 \\
\hline 22 & 24 & 0 & 250 & 100 \\
\hline 23 & 24 & 0 & 250 & 100 \\
\hline 24 & 12 & 0 & 500 & 100 \\
\hline 25 & 24 & 0 & 250 & 100 \\
\hline
\end{tabular}

Table 2: The data of OD.

\begin{tabular}{lccc}
\hline Category & Number & $\begin{array}{c}\text { Trip generation } \\
\text { and attraction }\end{array}$ & $\begin{array}{c}\text { Truncation normal distribution of trip } \\
\text { generation and attraction }\end{array}$ \\
\hline Trip generation site & 1 & $\overline{O_{1}}$ & $\mathrm{TN}\left(\overline{O_{1}}, \mu \overline{O_{1}}\right)$ \\
\hline Trip attraction site & 4 & $\overline{O_{4}}$ & $\mathrm{TN}\left(\overline{O_{4}}, \mu \overline{O_{4}}\right)$ \\
\hline
\end{tabular}

a existing road, and a dotted line is the symbol for a road to be built. Table 1 shows the basic information of the network, including free flow time, traffic capacity under the present situation, traffic capacity under planning situation, construction cost, and so forth. Table 2 is OD trip demand information, including deterministic demand and truncated normal distribution travel demand.

\subsection{Calculation Results}

This network is assumed to have three types of users, and the OD trip demand of each type of users submits to truncation normal distribution; time value is set to $0.5,1$, and 2 , respectively. 


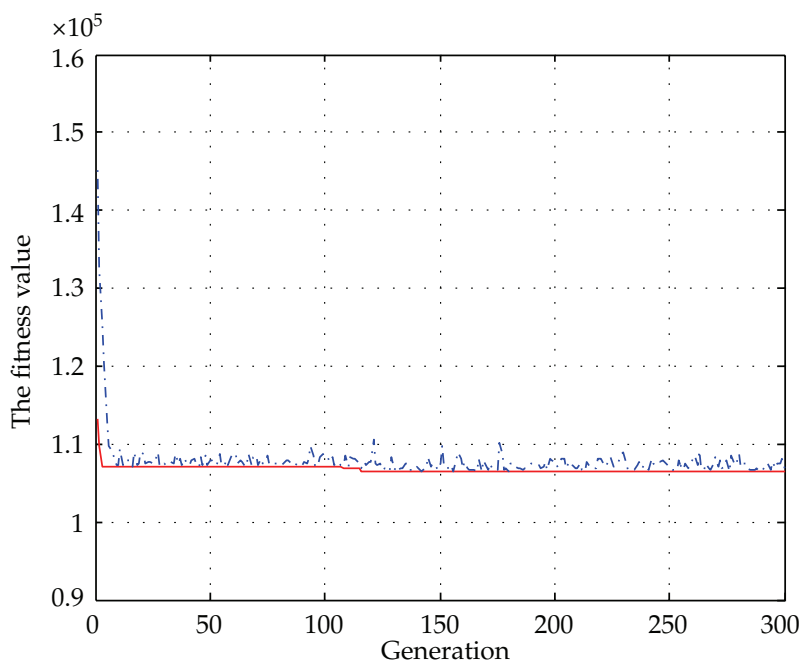

- The Minimum value

... The population mean

Figure 5: The solution of scenario II.

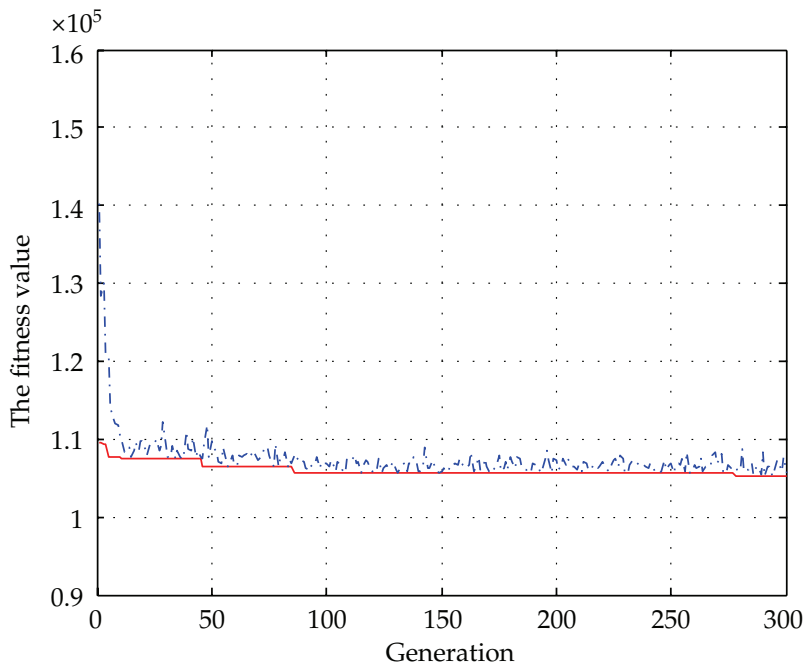

- The Minimum value

...- The population mean

Figure 6: The solution of scenario III.

There are 25 routes for new links and expanding links. So the parameters in genetic algorithm are set as the following. That is population size as 40 , evolutional generation range as 100, chromosome length as 25 , crossover probability as 0.8 , and mutation probability as 0.01 (Table 3). 


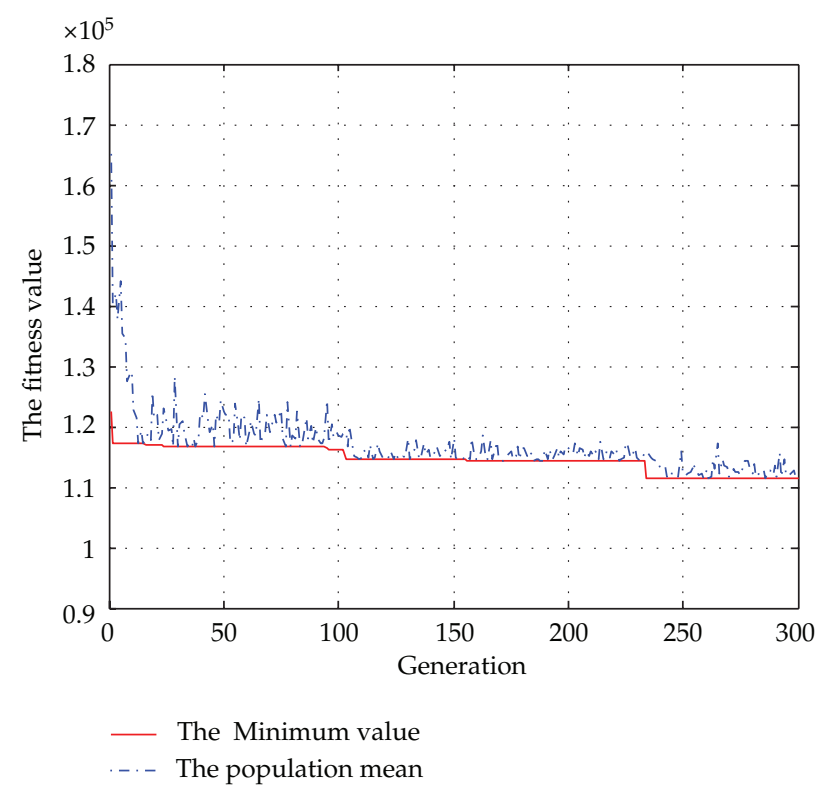

Figure 7: The solution of scenario IV.

Table 3: The parameters of the scenario.

\begin{tabular}{lccccc}
\hline Scenario & User demand & Mutation coefficient $\mu$ & Budget level & Risk coefficient $\rho$ & Sample size \\
\hline I & $100 / 150 / 100$ & 0 & 1000 & 1 & 1 \\
II & $50 / 250 / 50$ & 0 & 1000 & 1 & 1 \\
III & $250 / 50 / 50$ & 0 & 1000 & 1 & 1 \\
IV & $50 / 50 / 250$ & 0 & 1000 & 1 & 1 \\
V & $100 / 150 / 100$ & 0.2 & 1000 & 1 & 50 \\
VI & $100 / 150 / 100$ & 0.5 & 1000 & 1 & 50 \\
VII & $100 / 150 / 100$ & 0.2 & 1000 & 0.5 & 50 \\
VIII & 350 & 0 & 1000 & 1 & 1 \\
\hline
\end{tabular}

The evolution process under deterministic OD trip demand is shown in Figures 4, 5, 6 , and 7. The evolution process under uncertain OD trip demand is shown Figures 8, 9, and 10. The evolution process with only one type of users is shown in Figure 11.

Table 4 shows the calculation results of multiuser discrete transportation network under OD trip demand uncertainty, from which we can obtain the following conclusion.

(1) Scenario I and scenario VIII have the same total demand of all OD pairs, while scenario VIII has only one type of users and scenario I has three types of users. Results show that network planning scheme based on multiuser equilibrium model is different from that of single user model, and the total travel time of the system based on multiuser equilibrium model is higher.

(2) Contrasting from scenario I to scenario IV, OD trips of each type of users are different. The calculation results show that the OD trips proportion of different users to the total amount of the network has a significant impact on the planning scheme. In the transportation planning practice, the trip amount of different users 


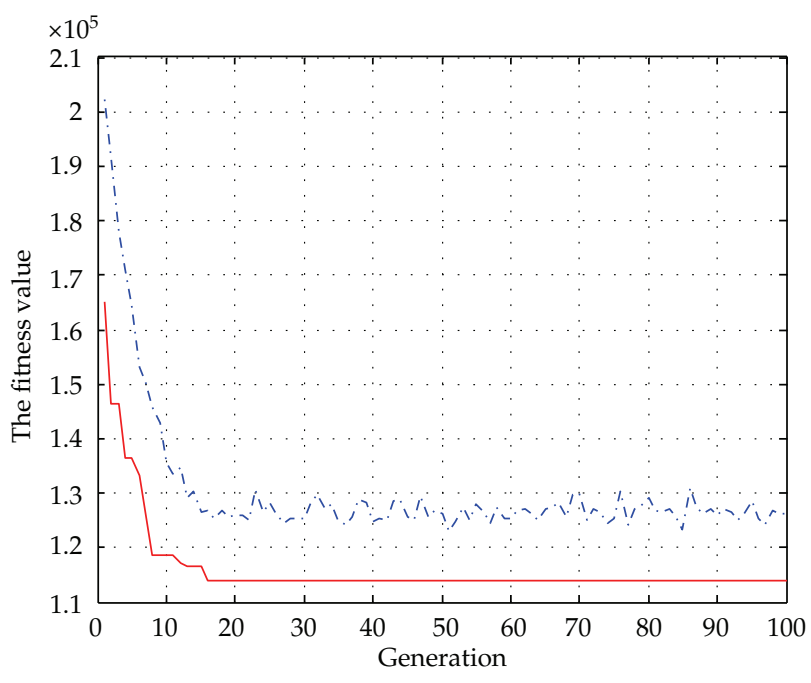

- The Minimum value

...- The population mean

Figure 8: The solution of scenario V.

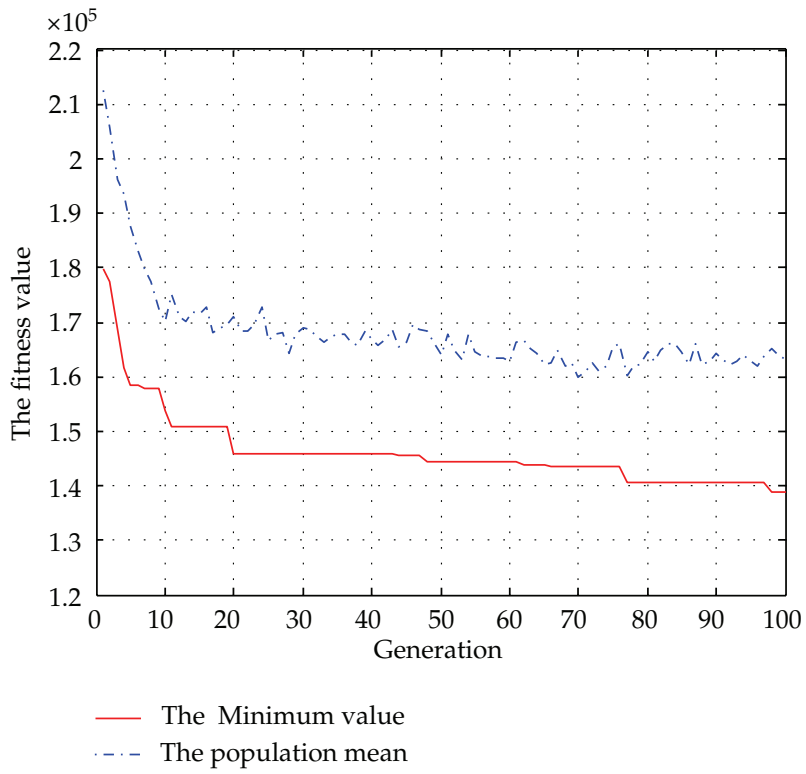

Figure 9: The solution of scenario VI.

should be determined according to social and economic characteristics of the region's inhabitants to provide a more powerful support for transportation network planning decision.

(3) Scenario V and scenario VI show the network planning results under the target function of system expected total travel time under OD trip uncertainty. It shows 


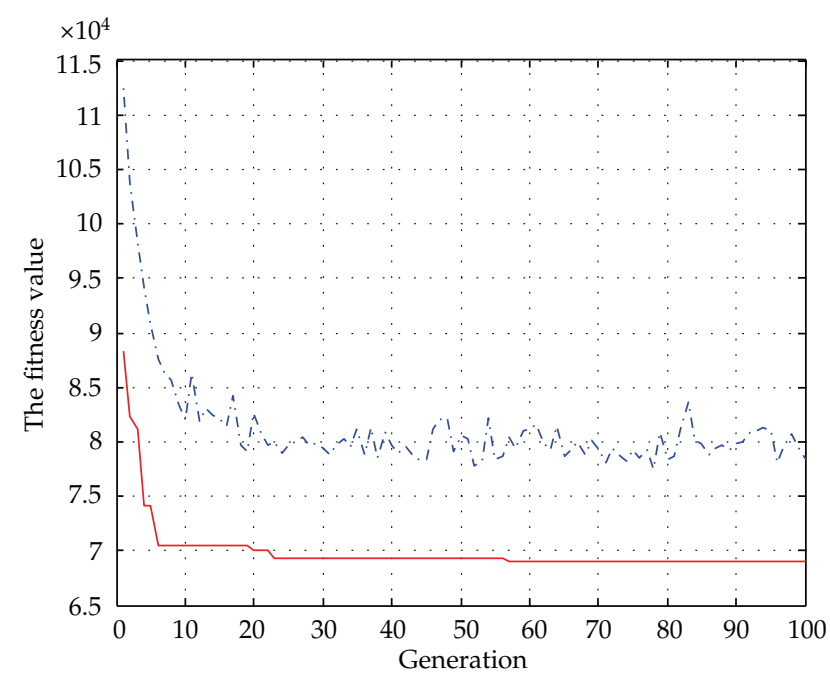

- The Minimum value

-.. The population mean

Figure 10: The solution of scenario VII.

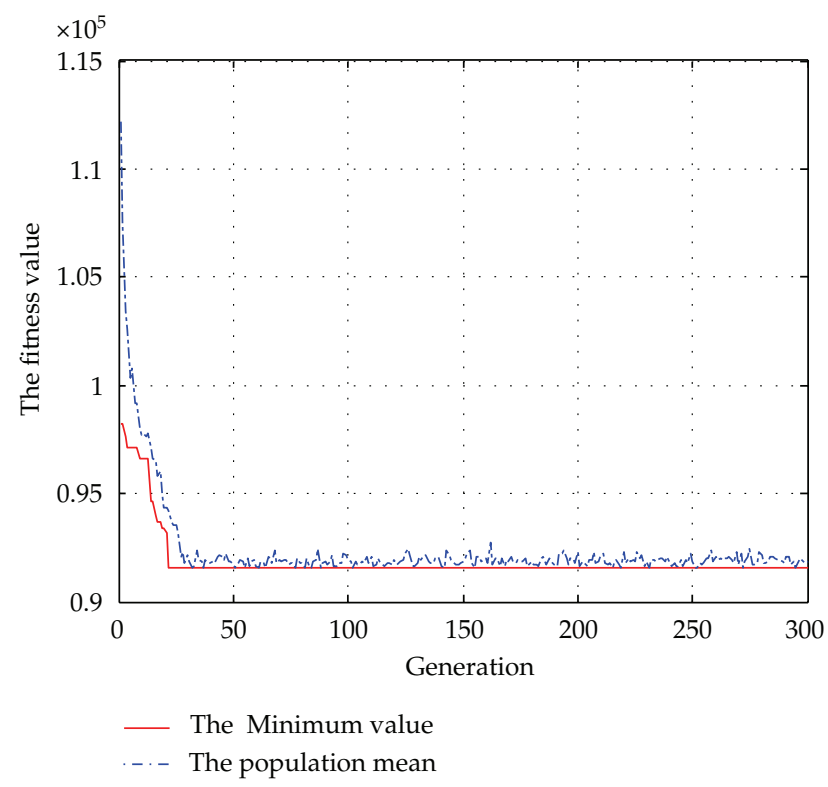

Figure 11: The solution of scenario VIII.

that the greater the OD demand uncertainty degree is, the greater the mean of the system total travel time is.

(4) Scenario V and scenario VII have the same degree of OD trip uncertainty. Risk preference of decision makers influences the final network planning scheme. 
Table 4: The solution of the sample.

\begin{tabular}{lcccc}
\hline Scenario & The fitter chromosome & Newly built roads & Extension roads & Fitness value \\
\hline I & 011101010110 & $21,22,23,25$ & $2,4,5,11,13,15$ & $1.05 * 10^{5}$ \\
& 0000101010000 & & & \\
II & 100101010100 & $20,23,25$ & $2,4,10,11,13,15,19$ & $1.07 * 10^{5}$ \\
& 0001101010001 & & & \\
III & 110101011100 & $20,21,23,25$ & $2,3,4,11,14,18$ & $1.05 * 10^{5}$ \\
& 0000100100010 & $22,23,25$ & $1,2,4,12,13,15,17$ & $1.11 * 10^{5}$ \\
IV & 001101110100 & & & \\
& 0000011010100 & 20,21 & $3,4,8,13,14,17,18,19$ & $1.14 * 10^{5}$ \\
V & 110000001100 & & & \\
& 0100001100111 & 23,25 & $2,4,6,10,11,13,14,18$ & $1.39 * 10^{5}$ \\
VI & 000101010101 & & & \\
& 0001101100010 & $20,22,23,25$ & $2,4,6,13,15,19$ & $6.91 * 10^{4}$ \\
VII & 101101010101 & 20,21 & $2,3,4$ & \\
& 0000001010001 & 22,23 & $13,14,15$ & $9.15 * 10^{4}$ \\
VIII & 111100011100 & &
\end{tabular}

\section{Conclusions}

In this paper, a discrete transportation network design problem is investigated, in which the trip generation flow and trip attraction flow are supposed as stochastic variables submitting to the given probability distribution. When travelers are divided into different groups by travel time value, a novel multiuser discrete network design model based on demand uncertainty is established. Genetic algorithm and Monte-Carlo simulation algorithm are integrated to solve the bi-level model for discrete network design. Calculation results on Nguyen Dupuis network show that user heterogeneity has a significant impact on network planning outcome under uncertain conditions. Furthermore, it can be found that GA is a potential tool for multiuser discrete transportation network design problem.

\section{Acknowledgments}

This work was supported by the Ph.D. Programs Foundation of Ministry of Education of China (20070003065), National High Technology Research and Development Program 863 (2007AA11Z202 and 2007AA11Z233).

\section{References}

[1] H. Farvaresh and M. M. Sepehri, "A single-level mixed integer linear formulation for a bi-level discrete network design problem," Transportation Research E, vol. 47, no. 5, pp. 623-640, 2011.

[2] E. Miandoabchi and R. Z. Farahani, "Optimizing reserve capacity of urban road networks in a discrete network design problem," Advances in Engineering Software, vol. 42, no. 12, pp. 1041-1050, 2011.

[3] M. Patriksson, "Robust bi-level optimization models in transportation science," Philosophical Transactions of the Royal Society of London A, vol. 366, no. 1872, pp. 1989-2004, 2008. 
[4] S. V. Ukkusuri and S. T. Waller, "Linear programming models for the user and system optimal dynamic network design problem: formulations, comparisons and extensions," Networks and Spatial Economics, vol. 8, no. 4, pp. 383-406, 2008.

[5] A. Karoonsoontawong and S. T. Waller, "Robust dynamic continuous network design problem," Transportation Research Record, vol. 2029, pp. 58-71, 2007.

[6] C. F. Daganzo, "Stochastic network equilibrium with multiple vehicle types and asymmetric, indefinite link cost jacobians," Transportation Science, vol. 17, no. 3, pp. 282-300, 1983.

[7] H. Yang, "Sensitivity analysis for the elastic-demand network equilibrium problem with applications," Transportation Research B, vol. 31, no. 1, pp. 55-70, 1997.

[8] Z.-C. Li, W. H. K. Lam, S. C. Wong, and A. Sumalee, "Environmentally sustainable toll design for congested road networks with uncertain demand," International Journal of Sustainable Transportation, vol. 6, no. 3, pp. 127-155, 2012.

[9] M. J. Smith, "The marginal cost taxation of a transportation network," Transportation Research B, vol. 13, no. 3, pp. 237-242, 1979.

[10] H. Yang and X. Zhang, "Multiclass network toll design problem with social and spatial equity constraints," Journal of Transportation Engineering, vol. 128, no. 5, pp. 420-428, 2002.

[11] R. B. Dial, "Bicriterion traffic assignment: basic theory and elementary algorithms," Transportation Science, vol. 30, no. 2, pp. 93-111, 1996.

[12] J. Agrawal and T. V. Mathew, "Transit route network design using parallel genetic algorithm," Journal of Computing in Civil Engineering, vol. 18, no. 3, pp. 248-256, 2004.

[13] B. Yu, Z. Z. Yang, and B. Yao, "An improved ant colony optimization for vehicle routing problem," European Journal of Operational Research, vol. 196, no. 1, pp. 171-176, 2009.

[14] B. Yu, Z.-Z. Yang, P.-H. Jin, S.-H. Wu, and B.-Z. Yao, "Transit route network design using ant colony optimization," Transportation Research C, vol. 22, pp. 58-75, 2012.

[15] B. Yu and Z. Z. Yang, "An ant colony optimization model: the period vehicle routing problem with time windows," Transportation Research E, vol. 47, no. 2, pp. 166-181, 2011.

[16] F. Zhao and X. G. Zeng, "Simulated annealing-genetic algorithm for transit network optimization," Journal of Computing in Civil Engineering, vol. 20, no. 1, pp. 57-68, 2006.

[17] S. B. Pattnaik, S. Mohan, and V. M. Tom, "Urban bus transit route network design using genetic algorithm," Journal of Transportation Engineering, vol. 124, no. 4, pp. 368-375, 1998.

[18] M. Bielli, M. Caramia, and P. Carotenuto, "Genetic algorithms in bus network optimization," Transportation Research C, vol. 10, no. 1, pp. 19-34, 2002.

[19] B. Yu, W. H. K. Lam, and M. L. Tam, "Bus arrival time prediction at bus stop with multiple routes," Transportation Research C, vol. 19, no. 6, pp. 1157-1170, 2011.

[20] B. Yu, Z. Z. Yang, and C. T. Cheng, "Optimizing the distribution of shopping centers with parallel genetic algorithm," Engineering Applications of Artificial Intelligence, vol. 20, no. 2, pp. 215-223, 2007.

[21] S. Nguyen and C. Dupuis, "Efficient method for computing traffic equilibria in networks with asymmetric transportation costs," Transportation Science, vol. 18, no. 2, pp. 185-202, 1984. 


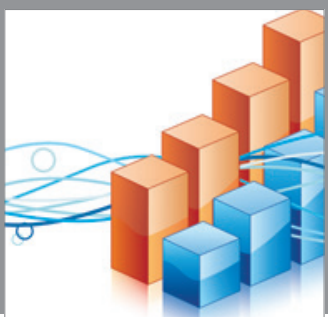

Advances in

Operations Research

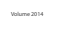

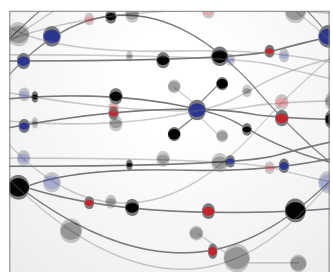

\section{The Scientific} World Journal
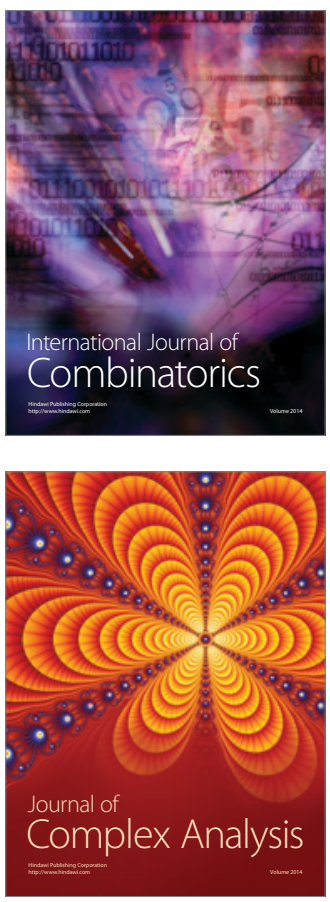

International Journal of

Mathematics and

Mathematical

Sciences
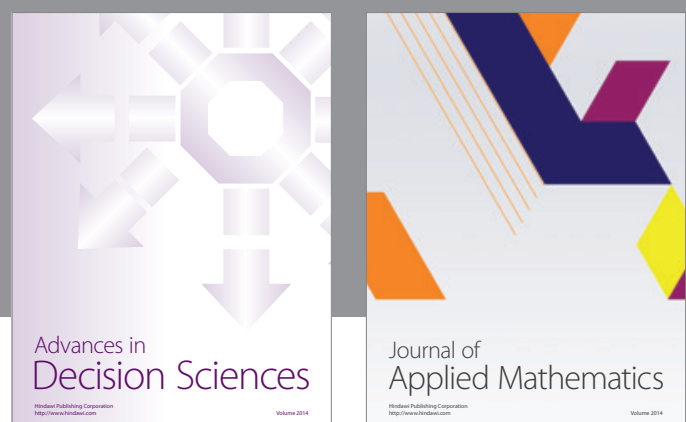

Journal of

Applied Mathematics
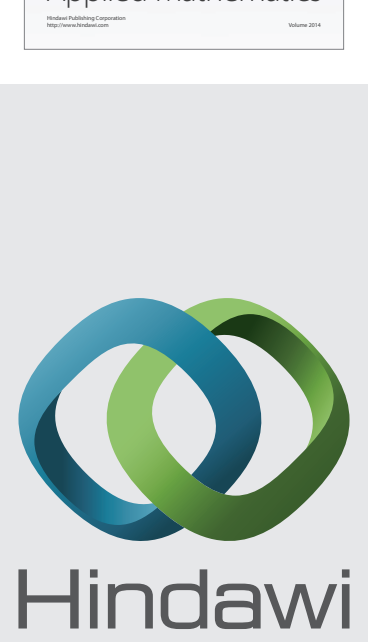

Submit your manuscripts at http://www.hindawi.com
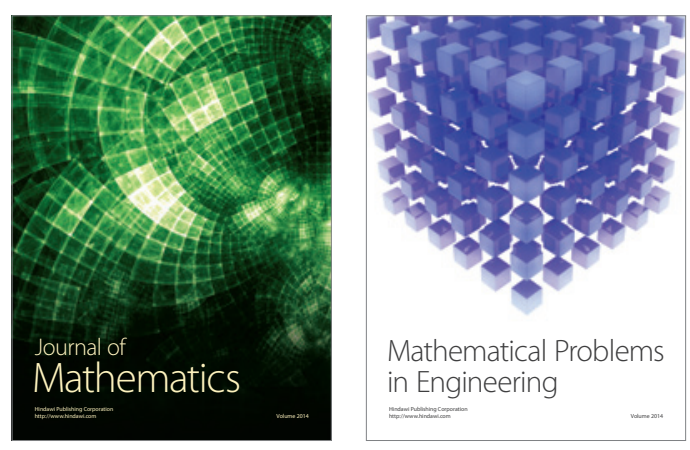

Mathematical Problems in Engineering
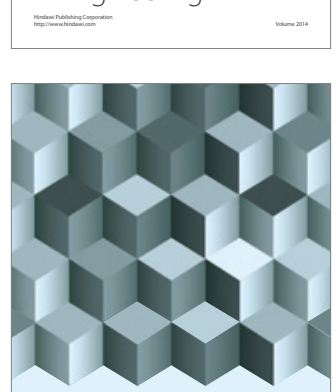

Journal of

Function Spaces
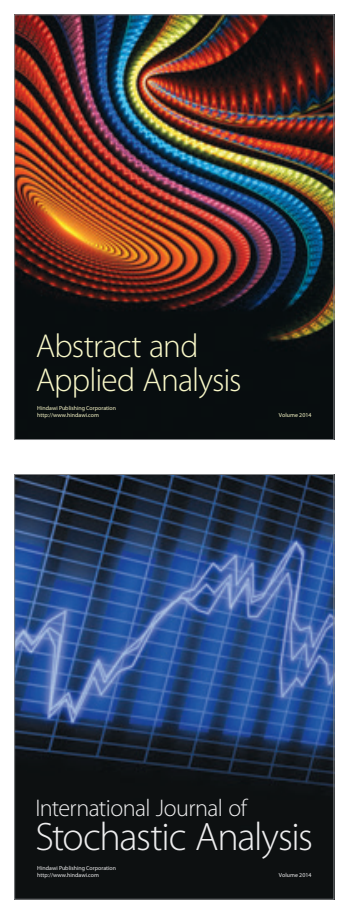

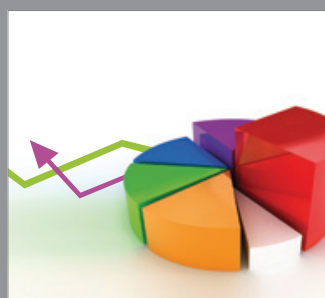

ournal of

Probability and Statistics

Promensencen
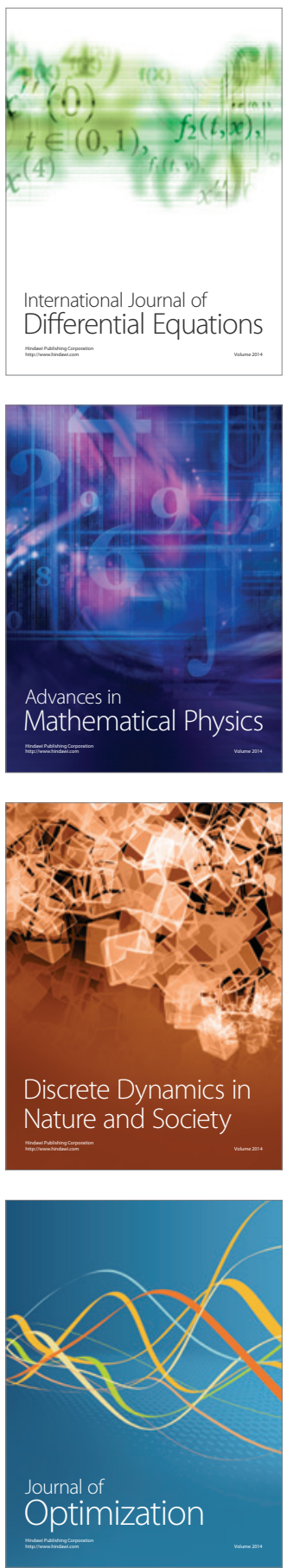